\title{
Niveles de acoplamiento y desacoplamiento en la relación familia - escuela en contextos rurales: el caso de una muestra de familias de Quispicanchis, Cusco
}

\author{
Martín Benavides \\ Heidi Rodrich \\ Magrith Mena
}

Grupo de Análisis para el Desarrollo (GRADE)

Martín Benavides es Doctor en Sociología y Magíster en Políticas Educativas por Pennsylvania State University. Ha sido estudiante visitante del área de movilidad social y desigualdad en Nuffield College en la Universidad de Oxford en Inglaterra. Es Director Ejecutivo e Investigador Principal de GRADE. Ha publicado sobre educación, estratificación y clases sociales, desigualdad, y juventud. Ha sido Fellow del Center for Advanced Studies in the Behavioral Sciences de la Universidad de Stanford durante el año 2007-2008.

Heidi Rodrich es Bachiller en Sociología por la Pontificia Universidad Católica del Perú. Ha sido Investigadora Asistente del área de educación de GRADE. Actualmente se encuentra iniciando sus estudios de maestría en el programa de Investigación de Educación en la Universidad Autónoma de Barcelona.

Magrith Mena es egresada de la Maestría de Ciencias Sociales con orientación en Educación por la Facultad Latinoamericana de Ciencias Sociales (FLACSO), Argentina y es Bachiller en Economía de la Pontificia Universidad Católica del Perú. Actualmente se desempeña como consultora en el área de educación de GRADE. 


\title{
Niveles de acoplamiento y desacoplamiento en la relación familia - escuela en contextos rurales: el caso de una muestra de familias de Quispicanchis, Cusco
}

\begin{abstract}
Resumen
Este estudio ha buscado analizar la relación entre dinámicas familiares y escolaridad, distinguiendo entre la participación de los padres de familia en actividades vinculadas a la escuela y los procesos de asignación de tiempo familiar a actividades educativas. El trabajo de campo se realizó con 80 familias de 10 centros poblados y comunidades de la provincia de Quispicanchis en el departamento de Cuzco. Se llevó a cabo un registro de actividades de los niños y sus familias -al inicio y durante el año escolar-, entrevistas y encuestas. Algunos de los principales resultados son que encontramos vínculos relativamente fuertes entre familias y escuelas en los aspectos más formales de la relación (principalmente en reuniones de Asociaciones de Padres de Familia) y que son los miembros de hogares que se comunican únicamente en quechua quienes menos participan. Sin embargo, por lo recogido en las entrevistas pareciera que, en términos generales, estas interacciones entre padres y profesores no se traducirían en herramientas claras que los padres puedan utilizar para influir positivamente en los procesos de aprendizaje planteados desde la escuela. En cuanto a la distribución del tiempo de los niños y niñas en actividades educativas fuera del horario escolar se encuentra, en general, que este es escaso y que en los casos de menor asignación esto parece asociarse a que los niños dedican una más alta proporción de su tiempo a las actividades laborales. Finalmente, se encuentra bastante heterogeneidad, aunque pueden distinguirse algunos patrones que apuntan a un mayor desacoplamiento con el sistema educativo formal de aquellas familias menos educadas, agrícolas y de zonas más altas.
\end{abstract}

Palabras clave: Uso del tiempo de los niños, Involucramiento familiar, Actividades educativas. 


\title{
Levels of coupling and decoupling in the family - school relationship in rural contexts: the case of a group of families in Quispicanchis, Cusco
}

\begin{abstract}
Summary
This study sought to analyze the relationship between family dynamics and schooling, distinguishing between the participation of parents in activities related to school and the within household allocation of time to educational activities. The fieldwork was done with 80 families of 10 communities in Quispicanchis, Cuzco. We register within household activities - at the beginning and during the school year-, and carried out interviews and surveys. Some of the main results are that we found relatively strong linkages in the more formal aspects of the family-school relationship. Also those families who communicate only in Quechua are less involved in school activities. We also found that the allocation of time to educational activities is relatively low and that less time allocated to educational activities seems to be associated to more time dedicated to labor activities. Also, we found quite heterogeneity in after school activities, although we can distinguish some patterns such as that those kids living in upper altitude communities with more labor activities have less time dedicated to educational activities.
\end{abstract}

Keywords: Children use of time, Parent involvement, Educational activities. 


\section{Introducción}

El presente estudio ha buscado analizar la relación entre dinámicas familiares

Ey escolaridad, profundizando en las características de la participación de los padres de familia en actividades vinculadas a la escuela y en los procesos de asignación de tiempo familiar a actividades educativas fuera del horario escolar. Se ha analizado por un lado en qué actividades participan las familias y por otro qué es lo que efectivamente implica en términos de la organización del tiempo y actividades familiares rurales el que sus hijos participen de la escolaridad formal.

\section{Orientación conceptual}

Debido a las múltiples dimensiones que componen el involucramiento familiar y a las distintas formas en que este ha sido medido (Fan \& Chen, 2001), es poco lo que se conoce sobre los contenidos del "efecto" de las familias, es decir sobre los mecanismos a través de los cuales los padres transmiten ventajas o desventajas educativas (Lareau, 2002, 2003). Se conoce aún menos sobre como esto se da en zonas rurales, o en contextos de mayor lejanía a los patrones urbanos de escolaridad (Benavides, Olivera \& Mena, 2006).

Sin embargo, la investigación ha hecho algunos avances, sobretodo con relación a la forma en que la pertenencia a determinado grupo social juega un rol en la definición del vínculo familias-escuelas. Ya hace más de dos décadas, Pierre Bourdieu (1986) señalaba que el determinante más fuerte para el logro escolar, pareciera ser la actitud familiar hacia la escuela, que se fija de acuerdo a los niveles, metas y expectativas del grupo al que se pertenece (Bourdieu 1986). Coleman (Coleman, 1988), resalta que dicho involucramiento o actitud de las familias es un aspecto del "capital social", que para él toma tres formas: obligaciones, expectativas y fiabilidad en el entorno social; canales de información; normas y sanciones efectivas. En esa línea Lee y Bowen (Lee y Bowen, 2006), encuentran efectos positivos entre el nivel educativo de los padres, el involucramiento educativo y el logro educativo. Por su parte, Larson y Verma (Larson \& Verma, 1999) ${ }^{1}$, resaltan diferencias en el uso del tiempo de los

1 En un análisis de varios estudios que se enfocan en el uso del tiempo de niños y adolescentes -entre 5 y 18 años- de diversos países (norteamericanos, europeos y asiáticos). 
niños y adolescentes entre poblaciones con distintas características ${ }^{2}$ : mientras los niños y adolescentes de sociedades "no industriales" dedican gran parte de su tiempo al trabajo (doméstico y/o remunerado) y a momentos de ocio "no estructurados", mayormente acompañados por miembros de su familia -nuclear o extensa-, aquellos pertenecientes a contextos "post industriales" ocupan su tiempo de maneras más variadas, dedicando la mayor parte a realizar tareas escolares y a actividades vinculadas a la tecnología o actividades extracurriculares (deportes, artes, etc.).

Posteriormente, Lareau (Lareau, 2002) propuso el concepto de los "patrones de crianza". La autora, tras un estudio empírico realizado en Estados Unidos, plantea diferencias entre "clases" con respecto a los diferentes roles de los padres en la educación de los hijos e hijas y sus diversas percepciones sobre "la naturaleza" de la infancia y niñez. Dicha autora encuentra patrones divergentes en el uso del tiempo, el lenguaje utilizado y las maneras de construir lazos, distinguiendo dos "lógicas culturales de crianza": la "crianza cultivada" (concerted cultivation) propia a las clases medias y "el crecimiento natural" (natural growth) característico a las familias "pobres" y de clase trabajadora. Esto de alguna manera es también afirmado por los estudios que analizan la comunicación entre padres e hijos, los cuales señalan que los niveles socioeconómicos influencian la relación entre comunicación y logro educativo (Park, 2008, refiriéndose a estudios de Ho Sui-Chu \& Williams, 1996; Desimone, 1999; McNeal 1999; y Lee \& Bowen, 2006).

Una de las áreas de investigación menos conocidas del vínculo entre familias y escuelas es aquella que analiza la forma cómo la institucionalización de los sistemas educativos afecta o no dicho vínculo. Hyujoon Park (2008) 3 trabaja la hipótesis de que los tipos de vínculos dependen de las características estructurales de los sistemas educativos, principalmente de sus niveles de estandarización. Así, los sistemas educativos menos estandarizados tendrían menores mecanismos de transparencia y rendición de cuentas y por ello para los padres resultaría más difícil tener una idea clara del proceso educativo, monitorear el aprendizaje de sus hijos o saber cómo ayudarlos. En los contextos en los cuales los padres de familia no conocen cómo ocurren los procesos escolares, cómo son los métodos de enseñanza y cómo utilizar esa información en las conversaciones con los hijos, habrá mayores barreras para la comunicación entre padres e hijos y consecuentemente menores logros de aprendizaje.

2 Por ejemplo, comparan poblaciones en contextos "no industriales" y "post industriales".

3 En un estudio comparativo realizado en 14 países. 
Estas barreras en la comunicación padres-hijos serían mayores para los padres y madres de niveles socioeconómicos bajos que para aquellos de niveles más altos, en tanto los primeros tendrían menos confianza para interactuar con los profesores y menor flexibilidad en sus horarios de trabajo (viéndose limitados para asistir a reuniones con los profesores o actividades de la escuela). El autor identifica que en los sistemas altamente estandarizados, la comunicación padres-hijos es más beneficiosa para aquellos estudiantes de niveles socioeconómicos bajos. Por el contrario, en el caso de los sistemas educativos no estandarizados, se encuentra una interacción positiva entre el nivel socioeconómico de las familias y la interacción padres-hijos ${ }^{4}$ (Park, 2008).

La menor o mayor estandarización de los sistemas educativos produce diferentes niveles de acoplamiento en la relación familias y escuelas: las sociedades que muestran un mayor acoplamiento entre la formalidad de la escuela, las prácticas de enseñanza y los rituales familiares, son aquellas donde el modelo de escolaridad se ha expandido coherentemente y donde no existe distancia entre los modelos de conocimiento y formas de organización del tiempo de la escuela y aquellos de las comunidades locales. Por el contrario, en aquellas donde los contenidos de la educación formal, su implementación por parte de los docentes y las culturas locales no comparten un modelo común, los niveles de desacoplamiento son mayores (Meyer, Scout \& Deal, 1983; Meyer, Boli \& Thomas, 1997). Estos no se expresan tanto en la formalidad del discurso o de los vínculos, sino más a nivel de los contenidos de las prácticas en la escuela y las familias.

Existen diversos estudios en el Perú que señalan que en los ámbitos rurales el sistema educativo se ha desarrollado de forma menos coherente o estandarizada. No solo se ha expandido más lentamente (Benavides, 2007), sino también que allí donde se ha logrado tener una escuela, la implementación curricular se da en menor medida (Galindo, 2002; Zambrano, 2004; Cueto, Ramirez, Leon \& Guerrero, 2004; Cueto, Ramirez, Leon \& Azañedo, 2006) y hay mayor evidencia de cierta readaptación de lo que se espera "oficialmente" que las escuelas lleven a cabo (Balarín, Benavides, Rodrich \& Rios, 2007). Esto último puede tener que ver con los conflictos entre el modelo de escolaridad, y los patrones culturales de enseñanza-aprendizaje, particularmente aquellos propios a las zonas rurales que se expresan en aspectos vinculados al uso de las lenguas, a los "saberes previos", a los métodos de enseñanza, etc. Estos conflictos constituyen una problemática vigente y han sido evidenciados en diversos estudios 
(Ruiz Bravo, Rosales \& Neyra, 2007; Balarín, Benavides, Rodrich \& Rios, 2007; Benavides, Olivera \& Mena, 2006; Trapnell, 2004; Ames, 2002; Ucelli, 1999).

Por ello, la hipótesis del estudio es que dado que en los ámbitos rurales la escuela se ha expandido de forma menos estandarizada, habrá mucha heterogeneidad en la relación familias escuelas, como resultado de las diferencias en los niveles de acoplamiento existente. Esa heterogeneidad se expresa más en los aspectos menos formales del vínculo familia y escuela y no puede ser fácilmente capturada por variables individuales, salvo por aquellas que puedan expresar más discontinuidad entre el modelo de escolaridad y las prácticas cotidianas de las familias.

\section{Muestra y metodología}

A partir de una base de datos de escuelas proporcionada por el proyecto EDUCAL se eligieron 80 familias ubicadas en 10 centros poblados/ comunidades de la provincia de Quispicanchis en el departamento de Cuzco5. La idea era contar con escuelas ubicadas en comunidades con distintas características geográficas: altas y bajas, rurales y urbanas ${ }^{6}$ pero que de acuerdo a los indicadores proporcionados tenían resultados educativos similares.

Los lugares seleccionados fueron Urcos, Quiquijana, Cusipata, Secsencalla, Kcauri, Pampachulla, Paucarpata, Ausaray, Yanama y Mujuncancha. De estos, la mitad se ubican en zonas altas y la mitad en zonas bajas y 7 de los 12 en zonas rurales. Además, durante la segunda visita incluimos a Andahuaylillas y Huaro, ya que cinco de los niños con quienes trabajábamos asistían a las escuelas de dichas zonas.

En un primer momento, se planteó realizar el trabajo de campo en dos etapas ya que cada comunidad debía ser visitada antes del inicio del año escolar o durante la primera semana de clases; $y$ a unos meses de iniciado el año escolar. Sin embargo, debido a las épocas de siembra y cosecha y a la cancelación de clases por fiestas patronales, el trabajo de campo se tuvo que realizar en tres visitas ${ }^{7}$.

5 Proyecto para el análisis de la calidad educativa escolar. Para mayor información ver: http://www. alter.org.pe/portal/home.php.

6 Aunque inicialmente se observaron 80 niños, solo 67 fueron considerados para el análisis, ya que los demás no pudieron ser observados durante las dos salidas o durante un tiempo suficiente.

7 La primera visita se realizó entre el 26 de Febrero y el 6 de Marzo del 2007 y en ella se trabajó en las 10 comunidades. La segunda visita se realizó sólo a las zonas bajas, entre el 16 y el 25 de 
La primera vez que se visitaron las zonas, la mayoría de colegios se encontraban en proceso de matrícula. Entonces, los observadores debían acercarse a la institución educativa, explicar al director el trabajo que iban a realizar y pedirle la lista de alumnos que se matricularían en cuarto grado de primaria. Los observadores debían dividir a los alumnos en dos grupos según su género y elegir al azar a cuatro alumnos de cada grupo. Luego, debían ubicar y presentarse con las familias de los alumnos seleccionados, explicarles el trabajo a realizar y conseguir su autorización para observar a los niños. De no poder ubicar a las familias o no conseguir la autorización de los padres para realizar el trabajo, los observadores debían reemplazar al niño seleccionado por otro de cuarto grado. De no poder trabajarse con un niño de cuarto grado, este debía reemplazarse por un niño de 5 to grado y de no ser posible con uno de 6to grado. El trabajador de campo debía observar a los niños durante 5 horas, entre las 2 p.m. y las 7 p.m.

Durante una misma salida, cada niño fue observado en dos oportunidades$^{8}$. Para ello se elaboró un cronograma según el cual los trabajadores de campo debían observar una vez a cada niño y luego hacer una segunda ronda de observaciones ${ }^{9}$.

Durante el trabajo de campo se aplicaron ocho instrumentos: Encuesta al padre/madre de familia; ficha de seguimiento del uso del tiempo del niño; ficha de observación del involucramiento familiar; inventario de las condiciones del espacio de estudio en el hogar; entrevista a los padres de familia; ficha de datos adicionales de la familia; ficha de información básica de la comunidad y guía de entrevista al director.

El instrumento central fue la ficha de observación del uso del tiempo del niño. En dichas fichas se detallaron y categorizaron todas las actividades que los niños realizaron durante las tardes en las que fueron observados. Asimismo se detalló si alguien (y quién) los acompañó durante estas actividades.

Mayo del 2007. En la tercera salida se visitó a las familias de las zonas altas y esta tuvo que ser realizada en dos partes (Julio y Septiembre) ya que cuando nos encontrábamos realizando el trabajo de campo comenzó la huelga de maestros.

8 Esto no fue lo que se planteó inicialmente, pero dado los cambios que hubo en el aumento de frecuencia de viajes por las razones explicadas anteriormente, por razones de presupuesto no se pudo concretar la idea de quedarnos una semana al menos. Esto puede generar un problema en el sentido que los niños son observados en días diferentes. Lo que se pudo controlar es que al menos a todos los niños les hayan dejado tareas el día de la observación y se consultó sobre el carácter típico de los dos días observados.

9 Durante todas las salidas a campo, el trabajo de los observadores fue permanentemente monitoreado por una supervisora, quien se encargó de homogenizar criterios, conocer las zonas y visitarlas constantemente; absolver dudas y reorganizar el trabajo de campo cuando ocurriera alguna situación no prevista. 
Las actividades realizadas por los niños fueron agrupadas en nueve categorías: escolares, recreativas, domésticas, descanso y aseo personal, económicas/ laborales realizadas en casa, económicas laborales realizadas fuera de casa, participación comunitaria, comidas y otros (se cambia, va al baño, se desplaza, etc.). Las actividades consideradas en la categoría "escolares" fueron: hacer tareas, estudiar o repasar, leer textos, leer revistas, periódicos u otros, y otra. Además, tuvo que incluirse una categoría en la que se agruparon actividades vinculadas a la presencia del observador (el niño observa, pregunta, o enseña algo al observador ${ }^{10}$ ).

Los demás instrumentos permitieron recoger información acerca de las características básicas de las comunidades, las escuelas y los hogares. Asimismo, se recogieron las opiniones y expectativas de los padres de familia y los directores con respecto al involucramiento familiar en la educación; la calidad de la educación de la zona y el funcionamiento de la APAFA y el CONEI ${ }^{11}$.

\section{Características principales de las comunidades, las escuelas y los niños observados}

Como puede verse en el apéndice, en términos de acceso y de dotación de servicios básicos y educativos se encuentran diferencias importantes entre las zonas bajas y altas. La zona de acceso más complicada es Mujuncancha. Para llegar ahí se toma un bus de Cuzco a Huaro que tarda casi dos horas, luego debe tomarse un carro particular durante una hora y media y finalmente caminar por aproximadamente 1 hora.

Como era esperable, es en las zonas altas y con mayores dificultades de acceso donde se cuenta con un menor acceso a servicios. Así, vemos que la escuela de Mujuncancha no cuenta con red de agua potable, desagüe o electricidad y que la de Ausaray tampoco cuenta con red de agua potable ni desagüe y que si bien tiene electricidad no cuenta con computadoras ni acceso a Internet. La escuela de Pampachulla, no es de acceso tan complicado pero se encuentra en un centro poblado relativamente pequeño y no tiene ninguno de los servicios. Asimismo, en cuanto al acceso a materiales educativos, es en las escuelas de las zonas más alejadas donde se identifican las mayores carencias.

10 Si bien los observadores tuvieron la consigna de no interactuar con los niños durante las observaciones, en algunos momentos (breves) estas situaciones no pudieron evitarse.

11 Asociación de Padres de Familia APAFA y Consejo Educativo Institucional CONEI. 
Según datos del 2004, las tasas más elevadas de extraedad se dan en el centro poblado de Kcauri y en las comunidades de Mujuncancha y Yanama, zonas rurales, de altura que se encuentran relativamente más alejadas de la ciudad del Cuzco y donde casi el $100 \%$ de los alumnos tienen como lengua materna el quechua. Por su parte, las tasas más altas de deserción, se dan en las comunidades de Mujuncancha y Secsencalla.

En cuanto a los niveles de rendimiento en comprensión lectora, vemos que estos son bastante bajos ya que es en los niveles "previo" y "menor al previo" donde se agrupan los mayores porcentajes de alumnos que rindieron la prueba ${ }^{12}$. Encontramos que es en las zonas rurales y más alejadas en las que se tienen los peores resultados. En Ausaray tenemos que el $100 \%$ de alumnos que rindieron la prueba se encuentran por debajo del nivel previo. En Kcauri este porcentaje es de $73 \%$ y en Yanama de $40.6 \%$. Además, en otras 4 zonas, la mayoría de ellas rurales, vemos que ninguno de los alumnos que rindió la prueba se encontraba en el nivel "suficiente".

En cuanto a las características de los niños observados, se tiene que de los 67 niños, poco más de la mitad son varones, y su edad promedio es 9.5 años, variando desde los 7 hasta los 12 años. Los grados que estos niños cursaron el año 2007 fueron el 4to, 5to o 6to grado de primaria, siendo el 4to grado de primaria al que asistieron la mayor parte de ellos (68,9\%).

En cuanto al acceso a materiales en el hogar y las condiciones de los espacios de estudio, encontramos que la mayor parte de los niños cuentan con los materiales básicos (lápiz, cuaderno, lapicero, borrador). Sin embargo, 29 de 67 no cuenta con ningún texto de lectura y 18 de 67 solo cuentan con un tipo de estos textos de lectura. ${ }^{13}$

\section{Resultados}

\section{La participación de los padres de familia en actividades escolares}

La mayor parte de los padres y madres ${ }^{14}$ de familia declara ir por lo menos una vez al mes (74\%) a visitar la escuela. Asimismo, un 42\% de padres afirma que

12 La prueba fue aplicada en Junio del 2006, a 99 alumnos de 40 escuelas de grupos de control y tratamiento del programa EDUCAL. Los resultados de la prueba se agrupan en 4 niveles: suficiente, básico, previo y menor al previo.

13 Los tipos de lectura considerados fueron libros de texto, cuentos, revistas, historietas, enciclopedias, novelas periódicos 
estas visitas se dan por iniciativa propia. No obstante, las visitas a la escuela también se hacen por invitaciones de los directivos de APAFA, del profesor o el director de la escuela. Por otra parte, las reuniones con los directivos de la APAFA resultan ser una práctica generalizada: el $94 \%$ de padres encuestados señaló que se reunió con algún directivo de la APAFA más de una vez durante el año 2006. Además, en las entrevistas, la mayoría de padres señalaron que las reuniones de APAFA se dan 3 o 4 veces al año.

Según lo recogido en las encuestas, la participación de los padres en la elección de representantes, la organización de alguna fiesta en la escuela y en reuniones por asuntos académicos o de disciplina suele ser bastante frecuente. La participación como parte del comité de aula o como directivo de la APAFA resulta menor: $52 \%$ de los padres declara no haber sido miembro del comité de aula y el $46 \%$ de ellos afirma no haber formado parte de la directiva de la APAFA.

En las entrevistas, los padres también señalaron que la forma más frecuente de participación de los padres y madres se da a través de la APAFA, mediante la realización de asambleas y faenas; y comités de aula. Sin embargo, muchos padres refirieron que hay quienes asisten a las asambleas porque se sienten "obligados" o por "temor a la multa" y que no todos los asistentes participan activamente en las mismas dando sus sugerencias o expresando sus preocupaciones.

Asimismo, en cuanto a formar parte de la directiva de la APAFA, los padres de familia indicaron que es difícil encontrar quienes quieran formar parte de la misma. Varios de los padres señalaron que es en la misma asamblea en la que se propone a un padre para que sea presidente de APAFA y que en muchos casos éste se siente comprometido y no puede negarse. A decir de los entrevistados, el que la mayoría de los padres de familia no quieran ser presidentes o parte de la directiva de APAFA se vincula principalmente a que ésta es una actividad para la que se requiere tiempo y/o recursos. Así, uno de los entrevistados, quien en ese momento era presidente de la APAFA de una de las comunidades, señaló que se le hacía difícil cumplir con sus responsabilidades como presidente y con su trabajo en la chacra.

El que los padres tengan poco tiempo para dedicarse a apoyar las actividades educativas de sus hijos fue constantemente mencionado (por padres de familia y directores) y aunque muchos señalaron que los padres si se preocupan por la educación de sus hijos, hubo comentarios de los propios padres que apuntan a que "a los demás padres no les interesa mucho la educación de sus hijos", que no se dan el tiempo necesario para apoyarlos, que "no están 
preparados" para asumir cargos o ayudar a sus hijos a hacer las tareas, etc. El ayudar a los hijos con sus tareas, por varios motivos, parece ser complicado para los padres de familia y algunos señalan que muchas veces esta "responsabilidad" recae sobre los hermanos mayores.

“¿Los papas acá ayudan a sus hijos hacer las tareas? A algunos les interesa a otros no ¿Y por qué cree que no les interesa?... algunos no saben leer, no pueden ayudar" (Madre de familia).

"Los padres de familia en lo que respecta a la parte pedagógica son indiferentes, ... . se les deja tarea pero los avances son mínimos. Los alumnos no tienen una hora para hacer tareas, después de los alimentos se dedican al pastoreo, a la chacra o asumen responsabilidades de los mayores, eso es una pena" (Directora de escuela).

\section{La participación y su asociación a las características de los hogares y sus miembros: participan más quienes más manejan los códigos culturales}

A partir de la información anterior se construyó un índice de participación de los padres y madres de familia en las actividades escolares de sus hijos. Este índice tiene un alpha de .68 y está compuesto por las siguientes variables ${ }^{15}$ :

- La participación de los padres como miembros de la directiva de la APAFA.

- La participación de los padres en la organización de fiestas en la escuela.

- La participación de los padres en la elección de representantes.

- La participación de los padres en reuniones de la escuela por asuntos académicos o de disciplina.

En esta sección se presentan los resultados de la aplicación de pruebas no paramétricas que buscan determinar a qué características de las familias se asocia una mayor o menor participación de los padres en las actividades escolares. Cabe subrayar, que la muestra es relativamente pequeña, y por ello

15 Se hicieron análisis de confiabilidad que llevaron a descartar otras variables para el índice: no se utilizaron por ser redundantes la frecuencia con que los padres visitan la escuela, el número de veces en que el padre o madre de familia se reunió con el directivo de la APAFA el año 2006, la visita a la escuela por iniciativa propia y la participación de los padres en algún momento como miembros del comité de aula. 
estos resultados son particularmente sensibles a la forma en que se agrupan las categorías de las variables ${ }^{16}$.

Los resultados de las encuestas señalan que los hogares en donde se habla castellano y quechua (o sólo castellano) participan más que aquellos en donde los miembros del hogar se comunican únicamente quechua. Con esto, la participación en las actividades escolares se estaría dando en mayor medida en un contexto de habla castellana, tal como los propios padres señalaban en las entrevistas.

Por otro lado, los padres que asistieron al menos a una capacitación sobre temas educativos, tienen una mayor participación en comparación con quienes no lo hicieron ${ }^{17}$. Finalmente, al observar la correlación entre la participación de los padres y el nivel socioeconómico de las familias, una primera aproximación nos dice que son los padres más pobres quienes más participan (con una correlación significativa al 10\%). Sin embargo, al agrupar a las familias en diferentes modos de acuerdo al número de NBI en el hogar, se tiene que esta asociación estadística no resulta robusta ${ }^{18}$.

\section{La asignación familiar del tiempo}

En el siguiente gráfico pueden verse los porcentajes promedio de tiempo que los niños observados dedicaron a realizar actividades escolares, recreativas, laborales o de otro tipo ${ }^{19}$, fuera del horario escolar.

16 Para efectos del análisis, se construyó una variable dicotómica que resume la participación de los padres de familia a partir del índice presentado anteriormente. Las variables correspondientes a las características de los hogares también fueron dicotomizadas.

17 Debe tenerse en cuenta aquí que el análisis efectuado no establece relación de causalidad. Por lo tanto, no puede concluirse que una mayor participación pueda estar determinada por una capacitación previa en asuntos educativos. Lo que aquí se comprueba es que existe una asociación entre estos dos hechos.

18 Dado que la correlación entre la participación y las notas del niño observado tuvo una significancia estadística cercana al $10 \%$, se decidió estimar las correlaciones con una forma alternativa de construcción de estas variables. Los resultados revelan que independientemente de cómo esta variable sea categorizada, las correlaciones entre ellas y la participación -aunque positivas- no resultan significativas. No tuvieron significancia estadística las siguientes variables: educación de papá, de mamá, número de hijos menores de 17, aspiración educativa para el hijo, notas del niño observado, al menos un hijo tiene extra edad.

19 En la Ficha de seguimiento del uso del tiempo del niño, las actividades agrupadas en la categoría otros fueron: Descanso y aseo personal, participación comunitaria y otros. Dentro de las actividades laborales se incluye aquellas realizadas dentro y fuera de la casa. 


\section{FIGURA 1}

Porcentaje de actividades realizadas en las salidas 1 y 2

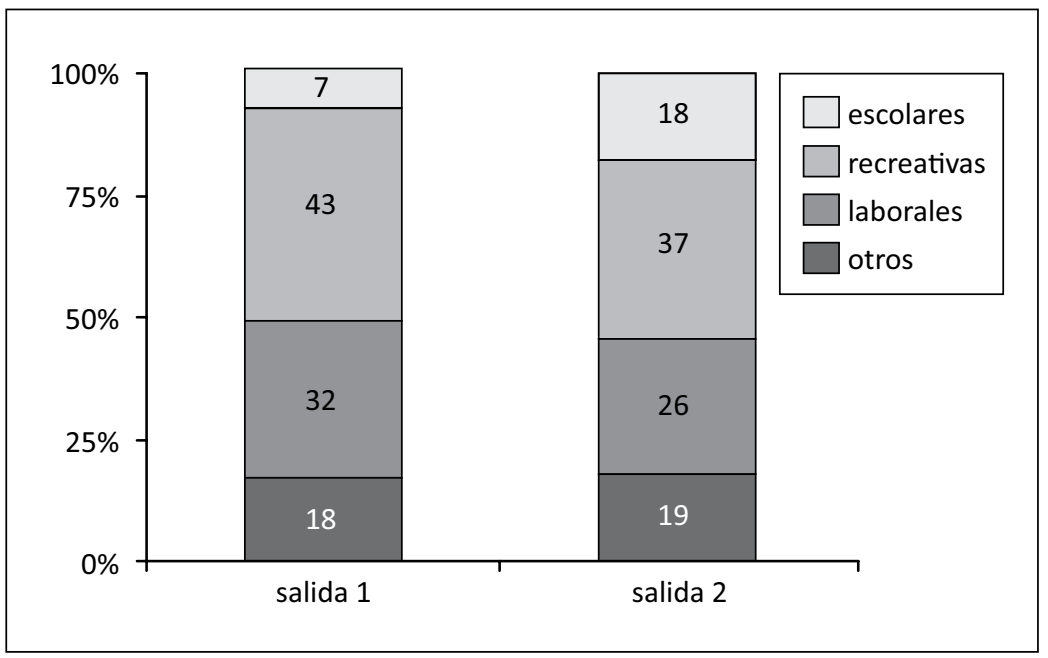

Como puede verse, en promedio, durante la primera salida (antes del inicio del año escolar) la mayor parte del tiempo observado fue dedicado a las actividades recreativas y laborales (el $75 \%$ del tiempo se reparte entre estos dos tipos de actividades), siendo las primeras a las que se dedica más del $40 \%$ del tiempo. Por su parte, las actividades escolares fueron las que recibieron -en promedio- la menor proporción de tiempo (7\%).

Durante la segunda salida, si bien se observan cambios en las proporciones de tiempo asignadas a cada tipo de actividad, la estructura de asignación sigue siendo la misma. Así, las actividades recreativas continúan siendo a las que se asigna una mayor proporción del tiempo (37\%), seguidas por las actividades laborales, aunque ahora la proporción de tiempo que se asigna a estas dos actividades (63\%) es menor que la observada en la salida 1 . Si bien encontramos que la proporción de tiempo asignado a las actividades escolares se incrementa del 7 al 18\%, este tipo de actividades continúa siendo a la que en promedio se asigna la menor proporción de tiempo. Y esto se da a pesar de que las clases en las escuelas ya se habían iniciado.

De igual modo, puede observarse que los incrementos en el tiempo dedicado a actividades escolares se asocian a una disminución de las actividades recreativas y laborales, mientras que la proporción de tiempo dedicado a las actividades clasificadas como otras se mantiene prácticamente estable. 


\section{FIGURA 2}

Actividades realizadas en las salidas 1 y 2 por tipo de zona

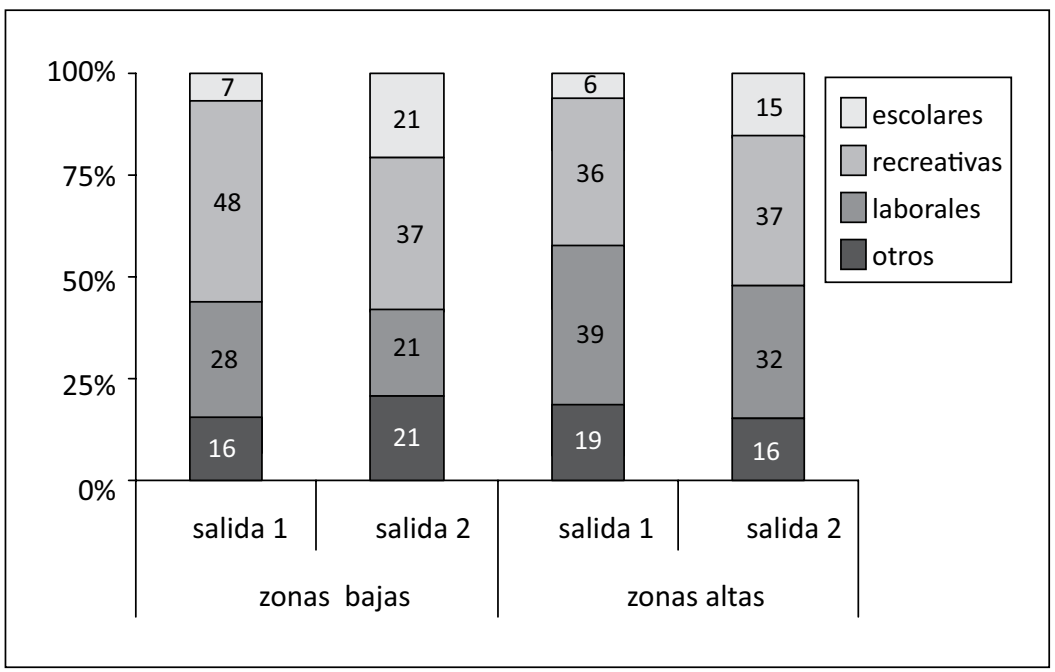

Al distinguir entre zonas altas y bajas, si bien la tendencia observada en el gráfico anterior se mantiene para los dos tipos de zona, encontramos importantes diferencias.

Así, aunque en la primera salida tanto en las zonas bajas como en las altas, la proporción de tiempo dedicado a las actividades escolares y otras es similar, no sucede lo mismo con el tiempo asignado a las actividades recreativas y laborales: en las zonas bajas, los niños dedican en promedio una mayor proporción de tiempo a las actividades recreativas y una menor a las actividades laborales. Así, mientras los niños de las zonas bajas pasaron casi la mitad del tiempo realizando actividades recreativas, los de las zonas altas dedicaron a este tipo de actividades un porcentaje menor (36\%). Asimismo, las actividades laborales ocuparon casi el $40 \%$ del tiempo observado para los niños de las zonas altas, y menos del 30\% para los de las zonas bajas.

Por su parte, los resultados de la segunda salida indican que en las zonas bajas se dio un mayor incremento del tiempo dedicado a las actividades escolares, llegando éstas a ocupar una proporción similar a las actividades laborales y otras. En las zonas altas, como dijimos, el incremento fue de menor magnitud y la proporción de tiempo asignado a las actividades laborales (aunque se redujo) duplicó la correspondiente a las actividades escolares. 


\section{La heterogeneidad de la asignación del tiempo}

Tal como mencionamos anteriormente, independientemente del tipo de zona en que residan, el tiempo que asignan los niños a las actividades escolares es muy variable, tanto en la primera como en la segunda salida.

Sin embargo, es posible formar grupos al interior de la muestra, de acuerdo al tiempo que los niños dedicaron a las actividades escolares durante la primera visita a campo, y a la magnitud del incremento o disminución que exhibieron en la segunda visita.

\section{Grupo 1: (36 miembros)}

Quienes dedicaron cero minutos a las actividades escolares en la salida 1 . Se tienen 4 sub-grupos:

- Grupo 1a. Quienes no incrementaron el tiempo dedicado a las actividades escolares (7 miembros).

- Grupo 1b. Quienes incrementaron el tiempo dedicado a las actividades escolares en menos de una hora pedagógica (45 minutos) (11 miembros).

- Grupo 1c. Quienes incrementaron entre una y dos horas pedagógicas el tiempo dedicado a las actividades escolares (11 miembros).

- Grupo 2d. Quienes incrementaron en más de dos horas pedagógicas el tiempo dedicado a las actividades escolares (7 miembros).

\section{Grupo 2: (15 miembros)}

Quienes dedicaron menos de una hora pedagógica a las actividades escolares en la salida 1. El grupo se divide en 3 sub-grupos:

- Grupo 2a. Quienes disminuyeron el tiempo dedicado a actividades escolares en menos de media hora pedagógica (5 miembros).

- Grupo 2b. Quienes incrementaron hasta una hora pedagógica el tiempo dedicado a actividades escolares. (8 miembros).

- Grupo 2c. Quienes incrementaron en una hora pedagógica o más el tiempo dedicado a actividades escolares ( 2 miembros).

\section{Grupo 3: (13 miembros)}

Quienes dedicaron una o más horas pedagógicas a las actividades escolares

en la salida 1. Al interior de este grupo se tiene 3 sub-grupos: 
- Grupo 3a. Quienes disminuyeron en más de una hora pedagógica el tiempo dedicado a actividades escolares (3 miembros).

- Grupo 3b. Quienes disminuyeron hasta en una hora pedagógica el tiempo dedicado a actividades escolares (6 miembros).

- Grupo 3c. Quienes mantuvieron o incrementaron el tiempo dedicado a las actividades escolares (4 miembros).

Tal como lo adelantábamos, si bien al interior del grupo 1 existe una relación entre mayor porcentaje de niños provenientes de zonas bajas, y mayores cambios positivos (incrementos) en el porcentaje de tiempo dedicado a las actividades escolares, esta relación no se mantiene para los grupos 2 y 3.

En cuanto al sexo de los niños, se tiene que en el grupo número 3 (niños que dedicaron más de una hora pedagógica a las actividades escolares), la mayor parte de integrantes son mujeres (77\%). En cambio, en los otros dos grupos, la mayor parte son niños. Sin embargo, al interior tanto del grupo 1 como del grupo 2, los sub-grupos donde se registraron los mayores incrementos en las actividades laborales tienen una alta proporción de niñas: $43 \%$ en el grupo 1 , y $100 \%$ en el grupo 2.

Por otra parte, en los grupos 1 y 2 , se observa una tendencia (no muy robusta) a que los mayores porcentajes de alumnos que estudiaron en la edad normativa, se encuentren entre los subgrupos donde se realizaron mayores incrementos en el tiempo dedicado a actividades educativas

En general, los niños del grupo 3 provienen de hogares relativamente menos pobres: en este grupo el número promedio de nbis es 0.5 (esta variable va desde 0 hasta 3), mientras que en los grupos 1 y 2 , la cantidad de nbis promedio asciende a 0.8 y 0.9 , respectivamente. En efecto, en el grupo 1 , en ningún caso los hogares tienen más de una nbi; mientras que en el grupo 2 , se encuentra hogares con hasta 3 nbis. No se encuentra que al interior de los grupos haya una relación entre menor número de nbis promedio y un mayor incremento del tiempo dedicado a actividades escolares.

En cuanto al nivel educativo alcanzado por los padres de familia en el sistema educativo formal, al interior del grupo 3 se observa un mayor porcentaje de padres que accedieron a la secundaria (92\%). En los grupos 1 y 2 , este porcentaje es $70 \%$ y $60 \%$, respectivamente. Lo mismo ocurre con la educación de las madres, en el grupo 1 se encuentra una mayor incidencia de madres que pudieron acceder al menos a la primaria, en comparación con los otros dos grupos. Al interior del grupo 1 se tiene una relación positiva entre mayor porcentaje de padres con educación secundaria y mayores incrementos; 
observándose esta relación también en el caso de la educación de la madre. Sin embargo, esta relación no se mantiene para los otros dos grupos.

Sin embargo, un resultado más claro es que en los subgrupos $2 c$ y $3 c-$ donde se registraron los mayores incrementos en la proporción de tiempo asignado a las actividades escolares en la segunda salida- no existen niños procedentes de hogares donde únicamente se hable quechua.

Si bien las variables individuales (salvo lengua) no permiten capturar tan claramente las diferencias relacionadas con el aumento de las actividades educativas entre un período y otro, si se puede identificar un grupo niños y niñas que se aleja bastante del resto de la muestra: los niños y niñas que no le dedicaron tiempo alguno a las actividades educativas.

Son siete los niños que en ninguna de las dos salidas dedicaron tiempo alguno a actividades escolares. A continuación algunos rasgos distintivos de ese grupo:

\section{TABLA 1}

Características de los siete niños que no dedicaron tiempo a actividades educativas

\begin{tabular}{cccccccc}
\hline Niño & Sexo & Grado & Edad & Zona & $\begin{array}{c}\text { Nivel educativo de la } \\
\text { madre }\end{array}$ & Lengua de la madre & $\begin{array}{c}\text { Número de } \\
\text { hermanos }\end{array}$ \\
\hline 46 & M & 6 & 12 & Secsencalla (rural-baja) & primaria completa & sólo quechua & 3 \\
64 & M & 4 & 8 & Kcauri (rural-alta) & primaria incompleta & quechua y castellano & 3 \\
71 & F & 5 & 9 & Paucarpata (rural-alta) & no estudió & sólo quechua & 3 \\
83 & F & 5 & 10 & Ausaray (rural-alta) & no estudió & sólo quechua & 5 \\
84 & F & 4 & 9 & Ausaray (rural-alta) & no estudió & sólo quechua & 5 \\
87 & M & 4 & 8 & Ausaray (rural-alta) & primaria incompleta & sólo quechua & 7 \\
94 & F & 4 & 8 & Yanama (rural-alta) & primaria incompleta & sólo quechua & 2 \\
\hline
\end{tabular}

Como puede verse en el cuadro anterior, los 7 niños provienen de zonas rurales, de las cuales la mayoría ( 6 de 7) son zonas altas. Asimismo, la mayoría de ellos pertenecen a hogares en los que la madre solo habla quechua, a diferencia del resto de la muestra donde la proporción de quechua hablantes es mucho menor. Para ese grupo, y a diferencia de los otros niños, las actividades recreativas no son las que muestran las mayores proporciones de tiempo asignado en la primera salida, sino las del tipo laboral. En seis 24 I de los siete casos, se tiene que las actividades laborales ocuparon entre el 
$27 \%$ y el $66 \%$ del tiempo observado, mientras que las recreativas recibieron como máximo el $38 \%$.

Durante la segunda salida, y nuevamente distinguiéndose del resto de la muestra, la proporción de tiempo que estos niños asignaron a las actividades laborales por lo general se mantuvo o incrementó, llegando a ocupar al menos la mitad de su tiempo, a diferencia de los otros casos. De esta manera, los datos sugieren que la escasez de tiempo asignado a las actividades escolares estaría asociada al hecho de que estos niños y niñas dedican una alta proporción de su tiempo a las actividades laborales, tanto en la primera como en la segunda salida.

\section{Reflexiones finales}

Este estudio implicó un intenso trabajo de campo y de posterior codificación de información. A pesar de ello y como se adelantó, no se pudo observar todo el tiempo que se había inicialmente planteado ( 1 semana entera por niño). Esto puede haber introducido el sesgo de observar en momentos diferentes a los niños: unos a inicios de la semana, otros al final de la misma. Sólo se pudo confirmar que todos tenían tareas. De otro lado, si bien las escuelas en conjunto son generalmente similares en términos de sus resultados, las familias puede que no lo sean. Por esto se recogieron datos de las mismas, aunque podrían estarse obviando otras diferencias.

Por ello, se buscó analizar información a nivel individual (niño por niño) para estar seguros de lo que se podía o no decir. Así, se puede señalar que la alta heterogeneidad encontrada en los aspectos relacionados al tiempo asignado a actividades educativas fuera del horario escolar contrasta fuertemente con la homogeneidad encontrada en las tasas de participación formal por la educación. En ese sentido se confirma aquí que se trata de procesos diferentes a través de los cuales se relacionan las familias con las escuelas. El hecho de que los padres participen de las Apafa puede no tener que ver con un seguimiento de estos a las actividades educativas de sus hijos fuera del horario escolar.

De otro lado, como se esperaba, es muy difícil capturar la heterogeneidad con las variables con las que se cuenta. Sólo la variable lengua señala una tendencia clara. Por ello, el análisis se orientó a buscar los casos más complicados y ver en que se diferencian del resto. Así se planteó el análisis de los 7 niños, quienes son fundamentalmente de las zonas altas y se dedican en mayor pro- 
porción a actividades laborales. Podríamos a partir de estos datos, sugerir que son las familias quechua hablantes, que habitan en la zonas altas y cuyos hijos dedican mayor tiempo a las actividades laborales, aquellas menos acopladas en términos relativos a la vida escolar, sea en su aspecto formal de participación como de involucramiento cotidiano. Esto confirmaría la hipótesis inicial de heterogeneidad y de mayor brecha familia escuela, en aquellos contextos de reciente escolarización y donde hay evidencia de menor implementación curricular y mayor conflicto entre saberes previos de las comunidades y los saberes de la escuela.

Estos resultados sugieren al menos dos cosas en términos de políticas: involucrar a los padres a través de la Apafa u otro mecanismo no tiene un impacto en la difusión de más actividades educativas al interior del hogar. Esa estrategia puede efectivamente tener una incidencia por el lado de empoderar a los padres, pero si no se acompaña de otro tipo de trabajo (como escuelas para padres) no tendrá resultados en términos de la mejora de la educación. Esto es particularmente importante para los casos de los hogares cuya lengua de uso predominante es el quechua, quienes participan menos y cuyos hijos dedican menos tiempo a actividades educativas.

Por otro parte, es claro que en el mundo rural existe un grupo de niños y niñas para quienes las oportunidades son efectivamente desiguales. Este grupo, que dedica poco tiempo a las actividades educativas, está compuesto por niños y niñas que trabajan más tiempo y que viven en zonas altas. Para ellos, si se trata de igualar oportunidades al menos con sus mismos pares rurales, debería buscarse una estrategia diferenciada de atención que tome más en cuenta la dinámica social y cultural de sus hogares, la cual- al menos en el caso de las familias de los 7 niños-le viene encontrando más sentido a que los niños se dediquen al trabajo, a pesar que simultáneamente valoran altamente la educación ${ }^{20}$.

20 Es decir, como pudimos observar a partir de nuestras entrevistas no se trata de un problema de una valoración baja de la educación como se suele señalar. 


\section{Referencias Bibliográficas}

Alternativa. Centro de Investigación Social y Educación Popular. (s. f.). Recuperado el 14 de enero de 2007, de http://www.alter.org.pe/portal/home.php

Ames, P. (2002). Para ser iguales, para ser distintos. Educación, escritura y poder en el Perú. Lima: IEP.

Balarín, M.; Benavides, M.; Rodrich, H; \& Rios, V. (2007). Prácticas docentes en colegios secundarios rurales. En Ministerio de educación, Estudio sobre la oferta y la demanda de educación secundaria en zonas rurales (pp. 173-317). Lima: MED.

Benavides, M. (2007). Lejos (aún) de la equidad: La persistencia de las desigualdades educativas en el Perú. En GRADE, Investigación, políticas y desarrollo en el Perú (pp. 457-483). Lima: GRADE.

Benavides, M.; Olivera, I.; Mena, M. (2006). De papás y mamás a hijos e hijas: las aspiraciones sobre el futuro y rol de las familias en las actividades escolares en el Perú rural. En M. Benavides (Ed.), Los desafíos de la escolaridad en el Perú. Estudios sobre los procesos pedagógicos, los saberes previos y el rol de las familias (pp. 154-211). Lima: GRADE.

Bourdieu, P. (1986). La escuela como fuerza conservador: desigualdades escolares y culturales. En P. de Leonardo (Ed.), La nueva sociología de la educación (pp. 103-129). México: Secretaría de Educación Pública.

Coleman, J. (1988). Social capital in the creation of human capital. American Journal of Sociology, 94 (1), 95-120.

Cueto, S.; Ramirez, C.; Leon, J. \& Azañedo, S. (2006). Oportunidades de aprendizaje y rendimiento en comunicación integral de estudiantes en tercer y cuarto grado de primaria en Lima y Ayacucho. En M. Benavides (Ed), Los desafíos de la escolaridad en el Perú: estudios sobre los procesos pedagógicos, los saberes previos y el rol de las familias (pp. 8-77). Lima: GRADE.

Cueto, S.; Ramirez, C.; Leon, J. \& Guerrero, G. (2004). Oportunidades de aprendizaje y rendimiento en matemática de los estudiantes de tercero y cuarto grados de primaria en Lima y Ayacucho. En M. Benavides (Ed), Educación, procesos pedagógicos y equidad: cuatro informes de investigación (pp. 15-67). Lima: GRADE.

Galindo, C. (2002). El currículo implementado como indicador del proceso educativo. En J. Rodríguez \& S. Vargas (Eds.), Análisis de los Resultados y Metodología de las Pruebas CRECER 1998. Documento de Trabajo 13 de MECEP (pp. 13-38). Lima: Ministerio de Educación. 
Lareau, A. (2002). Social class and childrearing in black families and white families. American Sociological Review, 67, 5, 747-776.

Lareau, A. (2003). Unequal childhoods: Class, Race, and Family Life. California: Berkeley.

Larson, R. \& Suman, V. (1999). How children and adolescents spend time across the world: work, play, and developmental opportunities. Pshychological Bulletin, 125 (6), 701-736.

Lee, J. S.. \& Bowen, N. (2006). Parent involvment, cultural capital, and the achievement gap among elementary school children. American Educational Research Journal, 43 (2), 193-218.

Meyer, J.; Scott, R. \& Deal, T. (1983). Institutional and technical sources of organizational structure: explaining the structure of educational organizations. En J. Meyer \& R. Scott (Eds.), Organizational Environments. Ritual and rationality, 45-70. New York, NY: Sage Publications.

Meyer, J.; Boli, J.; Thomas, G. \& Ramirez, F. (1997). World Society and the NationState. American Journal of Sociology 103, 144-81.

Park, H. (2008). The varied educational effects of parent-child communication: A comparative study of fourteen countries. Comparative Education Review, 52 (2), 219- 243.

Ruiz Bravo, P.; Rosales, J. L.; Neira, E. (2007). Educación y cultura: la importancia de los saberes previos en los procesos de enseñanza-aprendizaje. En M. Benavides (Ed.), Los desafíos de la escolaridad en el Perú. Estudios sobre los procesos pedagógicos, los saberes previos y el rol de las familias (pp. 73-156). Lima: GRADE.

Trapnell, L. (2004). Lenguaje y procesos de socialización: una aproximación crítica a los discursos y prácticas pedagógicas vigentes. Recuperado el 21 de agosto de 2009, de http://www.telecentros.pe/mazan/cronicas.shtml?x=8262

Uccelli, F. (1999). Educación y democracia en el sur andino: posibilidades y esfuerzos de las familia campesinas para educar a sus hijos. En M. Tanaka (Ed.), El poder visto desde abajo. Democracia, educación y ciudadanía (pp. 187-266). Lima: IEP.

Xitao, F. \& Chen, M. (2001). Parental involvement and student's academic achievment: a meta análisis. Educational Psychology Review, 13, 1-22.

Zambrano, G. (2004). Las oportunidades de aprendizaje en Matemática: un estudio para 4을 de secundaria. Boletín UMC 26 (pp. 1-20). Lima: MED. 


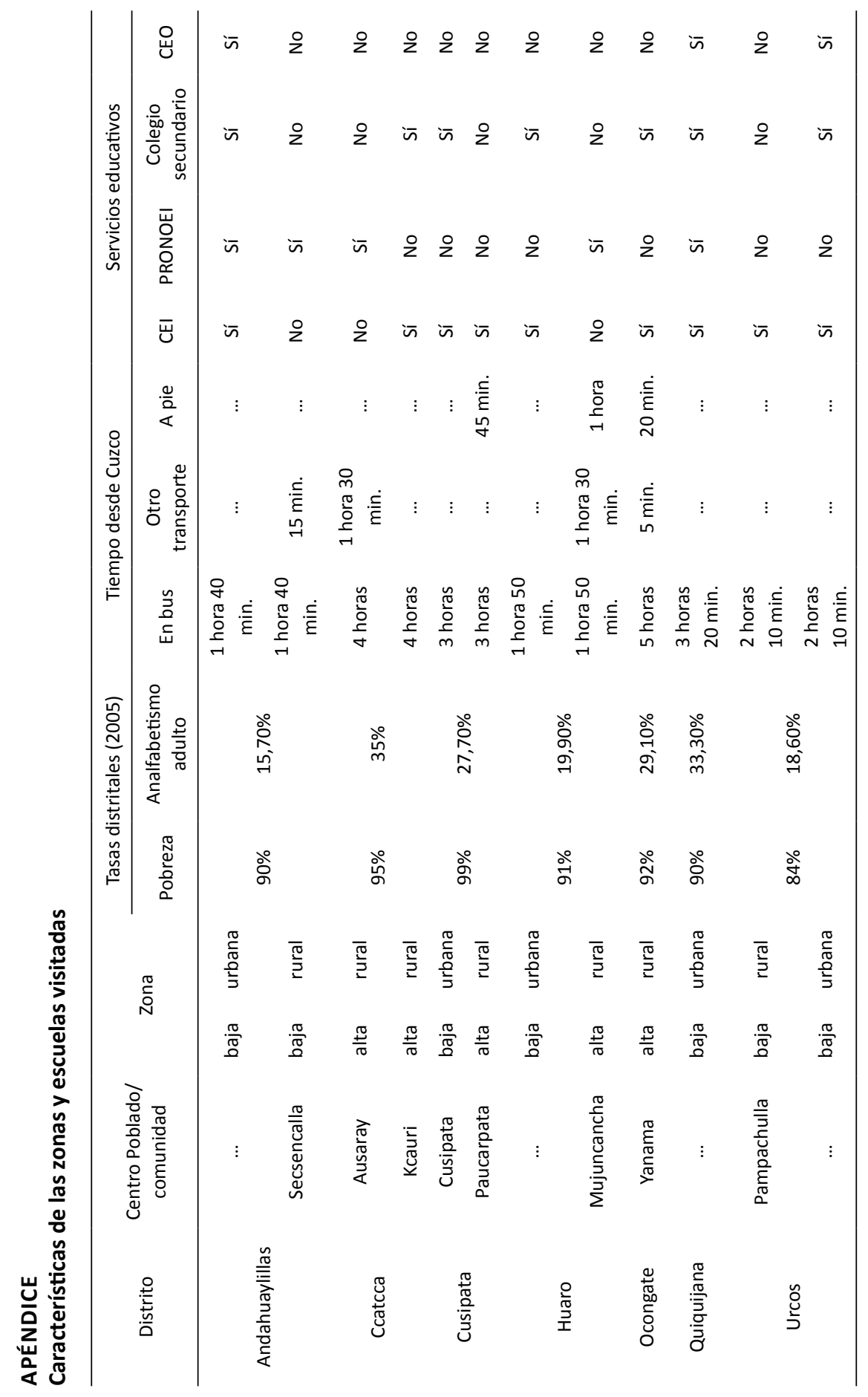




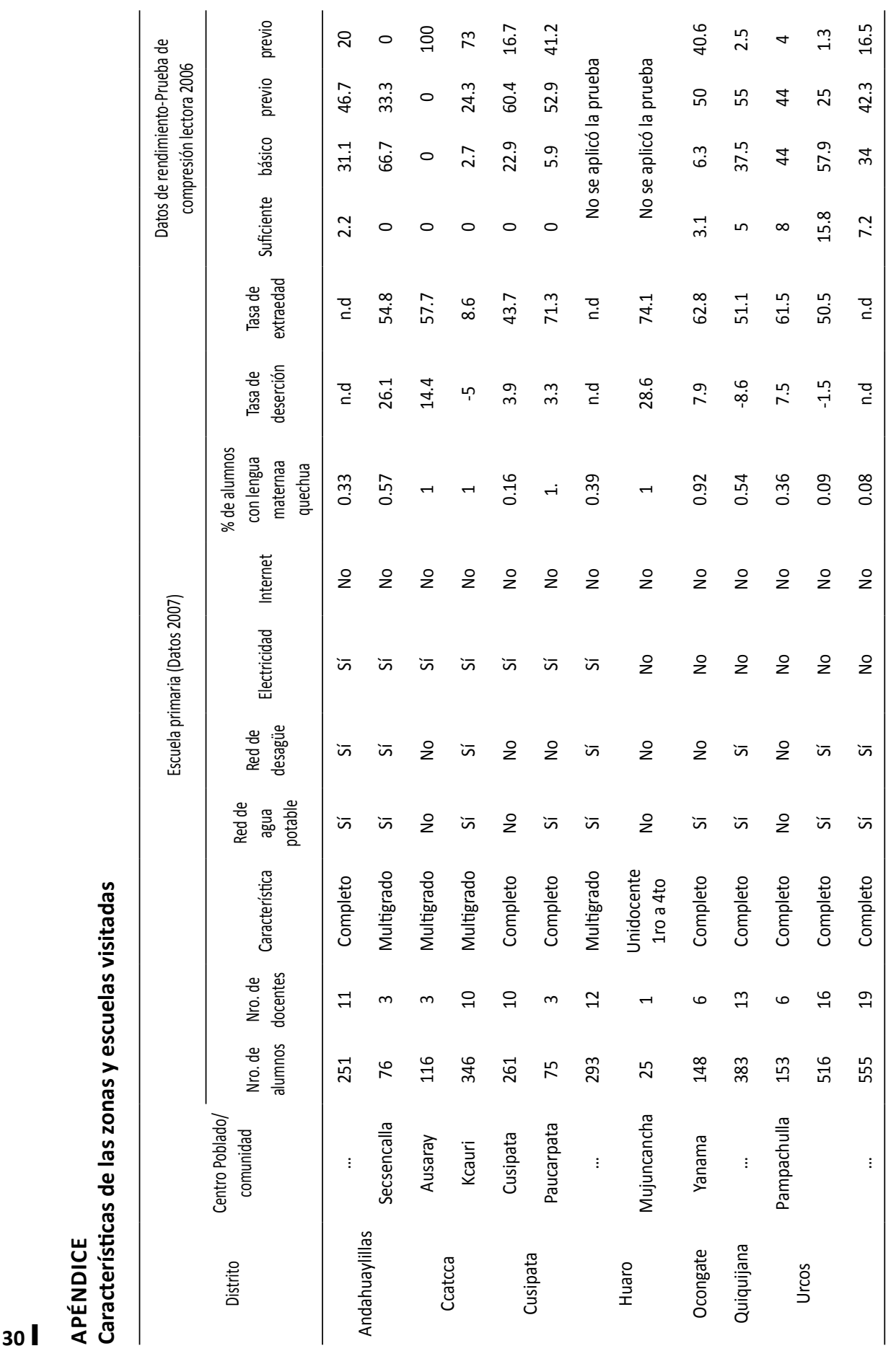

